\title{
Research opportunities in personalized cancer medicine
}

\author{
Advances in gene sequencing and targeted drug development \\ are helping make personalized healthcare a reality. What \\ does this mean for research careers in oncology?
}

\section{"The thing that's going}

\section{to distinguish people}

who get jobs and those

who don't is having

unique skill sets."

Hearn Jay Cho, New York University (NYU) Cancer Institute

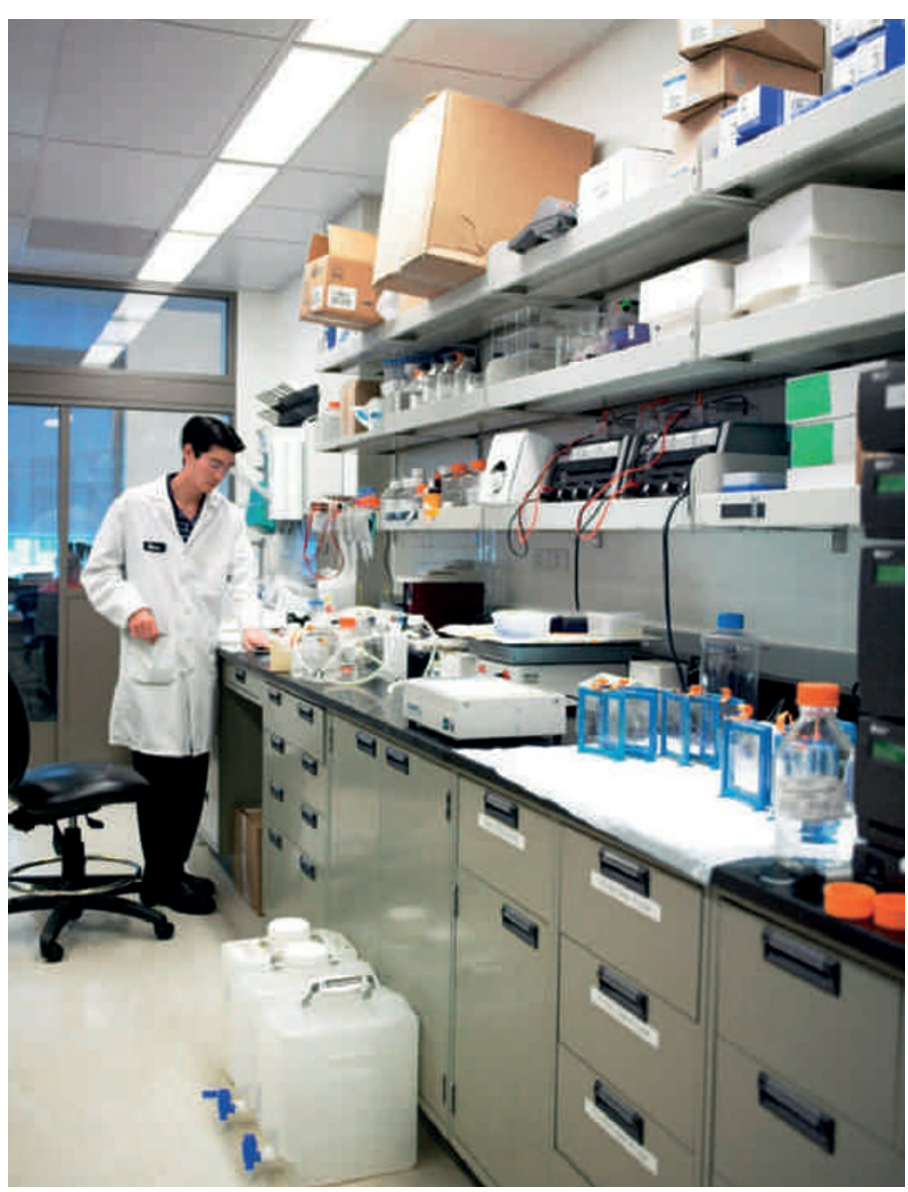

ONE OF the tantalizing possibilities of the Human Genome Project is the prospect of healthcare tailored to an individual's genetic profile — and the most tangible opportunity for personalized medicine is in the field of oncology. "A decade after the original publication of the draft human genome sequence, there are now incredibly exciting prospects for cancer patients," says Paul Workman, director of the Cancer

Research UK Cancer Therapeutics

Unit at the Institute of Cancer

Research (ICR) in the United

Kingdom. "Personalized medicine is already making a difference."

In personalized medical treatment, diagnostics can pinpoint which molecular abnormalities underlie a disease. Therapies designed to target those specific aberrations can then, in principle, provide maximum benefit for the patient with minimal effects on healthy cells. It's an approach that could revolutionize healthcare. "This is really the future of medicine," says Garret Hampton, senior director of development oncology diagnostics at California-based biotechnology company Genentech.

\section{A natural target}

Oncology is an ideal starting point for personalized medicine because cancer is essentially a blanket term for a multitude of disorders whose features are largely unique to each patient. For instance, when scientists at Washington University School of Medicine in St Louis, Missouri, and their co-researchers sequenced the genomes of breast tumours from 50 patients, they discovered the tumour's genetic mutations were highly diverse - of the 1,700 mutations discovered in total, most were unique to each patient's tumour, and only three mutations occurred in 10 percent or more.

"Cancer is a genetic disease, but one that can reflect changes in hundreds of genes," says Andrew Simpson, scientific director at the Ludwig Institute for Cancer Research (LICR), a global non-profit research organization with bases in Europe, the United States, Brazil and Australia.

"The permutations in any one particular tumour are rarely, if ever, reproduced between individuals."

However, some tumour characteristics are more common than others - for example, around one in five cases of breast cancer involve the over-expression of the HER2 protein, a characteristic that is targeted by the drug trastuzumab (Herceptin; Genentech). In April 2010 researchers from Stanford University's School of Medicine and their collaborators published a paper in Nature that showed around one-third of breast cancer patients have tumours that express high levels of an RNA transcript called HOTAIR.

The discovery of specific biomarkers and the development of targeted treatments and diagnostics requires a wide variety of researchers, says Wilbert Zwart, junior group leader at the department of molecular pathology at the Netherlands Cancer Institute (NKI). These include molecular biologists, cell biologists, geneticists, biochemists, pharmacologists and molecular imagers. The spectrum of disciplines provides the in-depth knowledge needed to evaluate a potential new treatment. "The success of a molecule in clinical development really depends on the extent to which one understands the mechanism of action a drug has against its target," says Genentech's Hampton, adding that a diagnostic test to correctly target the drug is another essential component.

A deluge of information is being 


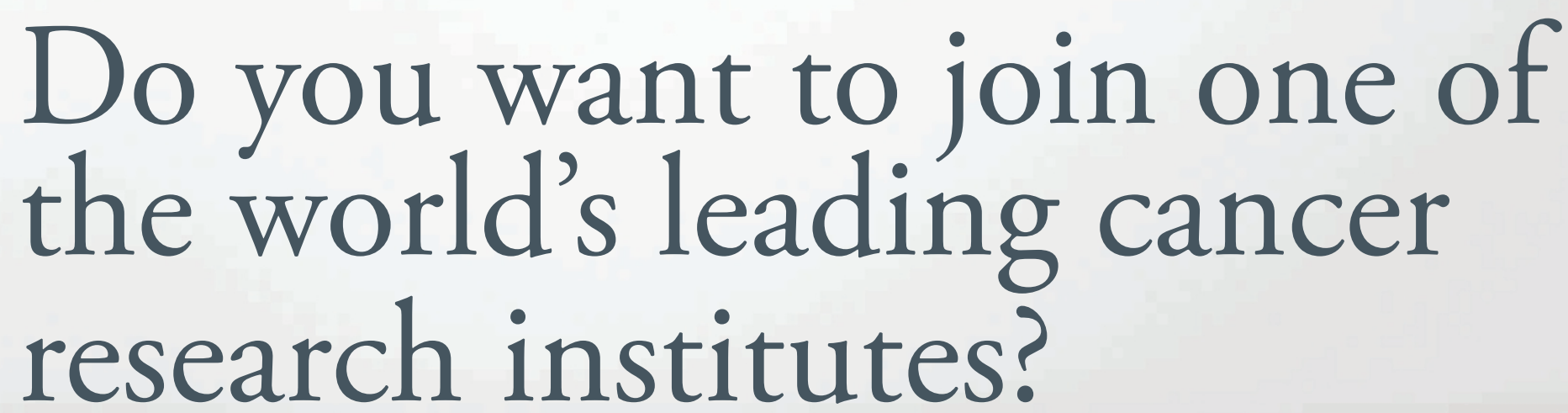

The Institute of Cancer Research is one of the world's leading cancer research organisations and has a long and distinguished history of research that has had a major impact on the outcome for cancer patients. With our partner The Royal Marsden NHS Foundation Trust we form the largest comprehensive cancer centre in Europe and perform high quality and original basic research and translational studies, with the aim of developing better treatments for the benefit of cancer patients worldwide. Under the leadership of our new Chief Executive Professor Alan Ashworth FRS we are committed to attracting, developing and retaining the best researchers in the world and are now looking to recruit exceptional scientists for senior positions in London and/or Sutton, Surrey, UK. Appointments may be made at tenuretrack equivalent or at a more senior level. We will provide appropriate support (including staff, consumables and equipment) for successful applicants' laboratories.

\section{Team Leader in Molecular Pathology}

Working with our partner the Royal Marsden NHS Foundation Trust we are at the forefront of advances in personalised therapy for cancer. To enhance our capacity in this area and to facilitate a rapid translation of basic research discoveries into benefit for cancer patients, we are building a dedicated Centre for Molecular Pathology.

We are looking for a new Team Leader to pursue an independent research programme investigating the molecular pathology of gastrointestinal tract cancers, with a particular emphasis on oesophageal and/or colorectal tumours.

\section{Team Leaders in Computational Genomics}

We are also looking for two team leaders in computational genomics to join the Centre for Molecular Pathology to facilitate the development of novel diagnostic, prognostic and therapeutic strategies by the analysis and meta-analysis of genomic data.

\section{Team Leader in Cancer Biology}

We are keen to recruit someone with expertise in the integration of multi-dimensional complex data to identify specific networks required for the survival of cancer cells, which builds on the Division of Cancer Biology's existing strengths in cell signalling.

If you are a strong candidate in other areas including proteomics, tumour microenvironment, metabolism and genetically engineered models of cancer, we would also like to hear from you.

\section{Team Leader in Cancer Genetics}

The Division of Genetics and Epidemiology has been exceptionally successful in identifying genes that predispose to cancer through linkage, GWAS and high-throughput sequencing strategies. We are now seeking to appoint a new Team Leader in genetics to utilize our excellent resources to contribute to the Division's focus on identifying genetic causes of cancer.

To apply for these posts you must hold a $\mathrm{PhD}$ in a relevant discipline or a closely related discipline and have an exceptional track record of research, demonstrated by your publications. Broad-based specialist knowledge in the appropriate area is essential as is the ability to co-ordinate, plan and execute research at an internationally competitive standard.

To apply for these exceptional posts, please visit http://www.icr.ac.uk/jobs/index.shtml Closing date: 31 October 2011 
generated by high-throughput sequencing of cancer genomes. This data flow will increase as the sequencing of patients' genomes and those of their tumours becomes a more affordable and integral aspect of standard care. National and regional screening programmes will contribute to the information flow. In the United Kingdom, for example, Cancer Research UK is leading a new Stratified Medicine Programme, with support from AstraZeneca and Pfizer, which will store DNA samples from the tumours of 9,000 cancer patients from the National Health Service (NHS) in a two-year pilot project. Mike Burgess, head of the oncology discovery and translational area of Roche's Pharma Research and Early Development organization, describes the breadth of opportunities that will arise as a result. He says people with skills in bioinformatics will obviously be vital to help make sense of the data, but expertise in mathematics and statistics will be invaluable.

\section{Multidisciplinary medicine}

Much of the data generation occurs in an academic or clinical setting and involves a wide range of disciplines. "We're following more than 80,000 patients right now and we're enrolling another 300 to 400 a week, so that's a lot of data," says William Dalton, chief executive officer and centre director at Moffitt Cancer Center in Tampa, Florida, which is storing the data for use in future studies. "The goal is to develop associations between different aspects of a patient's history and molecular data. We have major efforts in bioinformatics and information technology to provide quality data in a manageable way to clinicians, scientists, administrators and also patients - the effort will require a huge workforce," Dalton explains. They've also hired mathematicians "who've never touched a test tube" to work with colleagues in the lab to understand and model systems for better predictions.

Although researchers who are expert in their specialties are needed, so too are those "who have an overview and working knowledge of the whole spectrum of skills from gene to clinical trial," says ICR's Workman. "None of these activities are happening in isolation - each person has to work with colleagues in other areas, to help fit together the jigsaw puzzle of personalized medicine."

To this end, researchers with multidisciplinary expertise who can help translate research to the clinic are especially welcome in the field. For example physician-scientists scientifically trained clinicians who work in both patient care and research - are sought after. "There's definitely a market for MD-PhDs who can treat patients

\section{Top ten US institutes by National Cancer Institute (NCI) funding for projects involving genetic testing in 2010}

\section{Institute}

Harvard University School of

Public Health

University of Texas Health

University of Texas MD

Anderson Cancer Center

Mayo Clinic

Vanderbilt University

John Hopkins University

University of Pittsburgh at

Pittsburgh

St Jude Children's Research Hospital

$\mathrm{H}$. Lee Moffitt Cancer Center and Research Institute

Vanderbilt University
Science Center San Antonio

\section{Funding}

\section{$\$ 2,738,466$}

$\$ 2,512,500$

$\$ 2,452,446$

$\$ 2,300,000$

$\$ 2,300,000$

$\$ 2,233,067$

$\$ 2,156,287$

$\$ 1,876,655$

$\$ 1,863,711$

$\$ 1,594,760$
Project number

1U19CA148065-01

3U01CA086402-11S1

1U19CA148127-01

5P50CA102701-08

5P50СA090949-09

5P50CA088843-10

5P50CA090440-10

5U24CA055727-16

1U19CA148112-01

5U01CA114771-06
Source: NCI Funded Research Portfolio, http://fundedresearch.cancer.gov. Funding figures represent the proportion of total NCl funding that is related to genetic testing ('relevant funding')

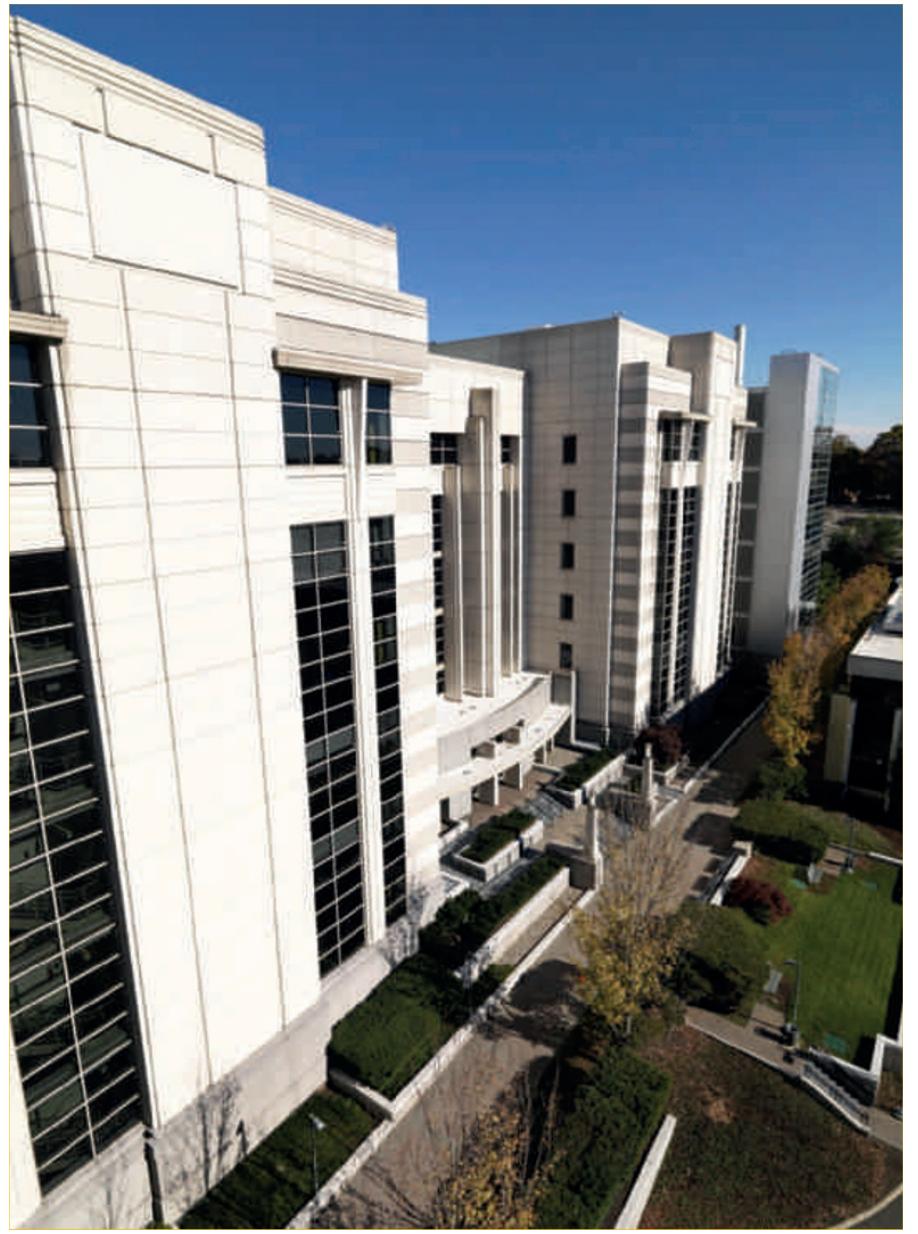

The main hubs of Roche's global R\&D network are located in Basel, Switzerland; Nutley, New Jersey, United States (pictured); and San Francisco, United States.

but also understand the genomics," Workman says. "Molecular pathologists are also really important — individuals who understand disease pathology in relation to different tumour types but also have a deep molecular understanding of the disease."

In the same vein, a collaborative spirit among researchers is crucial. “We don't need people working in their own ivory towers - we need the kind of personality that can work and communicate with others, understand what contributions they bring to the table, and seeks diverse talent," says Dalton of Moffitt Cancer Center. "We need team players."

Collaborations between institutions will also be essential for the development of personalized medicine. Because the targeted therapies are specific to relatively small groups, researchers may need to form consortia to find enough patients to recruit for studies. Dalton highlights an example of this at Moffit Cancer Center: "We had a new drug that was being tested in metastatic colorectal cancer, and we found 37 patients in three and a half months using our database, which pools together information from 18 partners in 10 different states." He and his colleagues soon hope to include international partners in the database.

\section{Developing targeted drugs} As well as research collaborations, personalized medicine for cancer will necessarily involve combinations of drugs specialized therapies might only be useful to combat one aspect of what are often very complicated cancers. "Many drugs will be available that may not be really effective on their own, so there will be a lot of work available in analyzing what combinations are effective," says LICR's Simpson. 
을 $\quad$ And, while a single drug might prove effective against a cancer for a set period, resistance to that drug may later develop. The solution to this may also be a combination of therapies. "It's like chess - we have to anticipate whatever move a cancer might make and counteract it before it adapts," Dalton says. This approach will provide opportunities for those working in the relatively new field of systems biology. "You want systems biology expertise to help understand how perturbations in one system might cause changes in another," Dalton explains.

The move toward treatment combinations will lead to new strategies for clinical trials. "You'll see multiple small trials [running] together to accomplish more," says Anna Pavlick, director of the melanoma programme at the New York University (NYU) Cancer Institute. "For instance, after you prove safety with a single therapy in a phase I trial, as it moves into phase II you'll combine that therapy with others in another phase I trial."

Novel therapies are not the only focus of personalized medicine. Any treatment requires complementary diagnostics to pinpoint which specific patients it will suit best. As well as an important factor in designing diagnostics. "You want rapid turnaround time - sometimes patients may not have three weeks [for you] to run a test," Pavlick explains. "There's a desire for researchers to develop assays sensitivity and precision, speed is that work quickly." To explain Pavlick points to a diagnostic test for vemurafenib (Zelboraf; Roche/Plexxikon), a drug that received approval from the US Food and Drug Administration (FDA) in August this year for use in patients with inoperable or metastatic melanoma that carries the BRAF V600E mutation. The test, called the cobas BRAF Mutation Test, is PCR-based and will indicate whether a patient is suitable for treatment with vemurafenib within 72 hours.

Given the move toward niche drugs that target smaller populations of patients, LICR's Simpson sees plenty of opportunities for very small companies that each develop a single reagent. "You'll see these 'micro-biotechs' designed as rather temporary things, forming and closing as they either bring drugs through trials and license them to a larger entity or see failure of that particular reagent," he says. "There'll be real demand for people with a mixture of drug-making skills and business skills to run those companies.”

\section{An international job market}

Although there are opportunities for a wide range of disciplines in personalized medicine, the sector is subject to the tight job market affecting all others. Despite that, there are ways to stand out from the crowd. "The thing that's going to distinguish people who get jobs and those who don't is having unique skill sets, such as

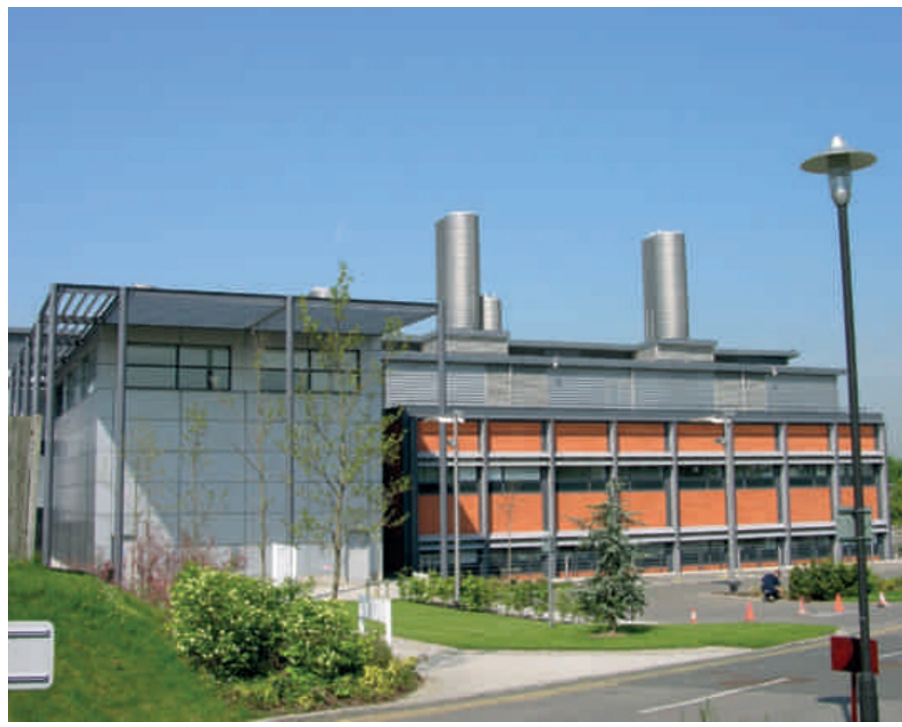

Around 1,000 staff and postgraduate students work for the UK's Institute of Cancer Research (ICR). information technology, computer science and systems engineering in addition to understanding biology," says Hearn Jay Cho, assistant professor of medicine and pathology at the NYU Cancer Institute. "That will really make people competitive."

Employment possibilities in personalized cancer healthcare United States and Europe. "China is a big growth area for this type of expertise [and it] is going to expand exponentially - they have a large population and they have the infrastructure necessary to analyze these types of data sets," Roche's Burgess says. research are not restricted to the

\section{Top ten cancer research projects funded by the EU between 2007 and 2009}

\section{Project}

1 INFLA-CARE

3 COGS

4 EUCAAD

5 O3K

7 SKINSPECTION

8 LOULLA\&PHILLA

$9=$ ADAMANT

$9=$ MISMATCH2MODEL

$9=$ TARCC

10 CANCERDIP
2 METOXIA

6 FLUODIAMON

\section{Coordinator and institute}

Dr Aristides Eliopoulos, University of Crete, Greece

European Commission funding

Dr Erik Pettersen, University of Oslo, Norway

Dr Per Hall, Karolinska Institute, Sweden

Dr Tina Dalianis, Karolinska Institute, Sweden

Dr Gilles Vassal, Institut Gustave Roussy, France

Dr Jerker Widengren, KTH Royal Institute of Technology, Sweden

Dr Robert Lemor, Fraunhofer Society, Germany

Dr Vincent Grek, Only For Children Pharmaceuticals (O4CP), France

Dr Raffaella Giavazzi, Mario Negri Institute for Pharmacological Research, Italy

Source: Cancer Research EU Funding (2007-2009), European Commission

Dr Manel Esteller, Bellvitge Institute for Biomedical Research, Spain
$€ 11,999,889$

$€ 11,998,300$

$€ 11,715,501$

$€ 5,989,862$

$€ 5,958,419$

$€ 4,197,774$

$€ 4,097,585$

$€ 3,316,415$

$€ 3,000,000$

$€ 3,000,000$

$€ 3,000,000$

$€ 2,999,994$ Dr Jacques Barbet, National Institute of Health and Medical Research (INSERM), France
James Christensen, director of translational pharmacology at Pfizer, concurs. "Pfizer is doing more and more work in Asia, particularly China - we're working with academic centres and contract research organizations there on drug discovery," he says. "There's also interest there in understanding the molecular basis of common malignancies in Asia, such as hepatocellular carcinoma and gastric cancer."

Personalized cancer medicine is also advancing rapidly in Australia, says Pavlick of the NYU Cancer Institute, also pointing to development in the Middle East. "There's a huge push right now to build science in Dubai," she says. NKI's Zwart mentions Singapore as another country advancing in this field and attracting very skilled scientists. Simpson from LICR says that the potential for micro-biotechs in the coming decade will make it easier for contributions from developing countries where drug development is not currently well-established such as Latin American countries.

As the foundations of personalized medicine are laid across the world, the likelihood increases that the Human Genome Project will benefit all of humanity in the fight against cancer. "This field is really becoming global," Burgess concludes.

Nature editorial staff have no responsibility for content 


\section{The Beatson Institute for Cancer Research Postdoctoral Research Scientists}

institute for cancer research

Starting Salary from $€ 28,500$ to $£ 38,000$ (Depending on experience)

Initial 3 year fixed term contract

The Beatson Institute in Glasgow is one of Europe's leading cancer research centres. It is core funded by Cancer Research UK and supports cutting edge research into the molecular mechanisms of cancer development. The Institute provides an outstanding research environment, underpinned by state-of-the-art core services and advanced technologies with special emphasis on imaging and proteomics.

\section{Vascular proteomics - Dr Sara Zanivan}

The Vascular Proteomics group is a growing team with a major interest in tumour angiogenesis. You will join our effort to better characterise endothelial cells in vivo and profile molecular mechanisms regulating angiogenesis in normal and tumour conditions using in vitro models. In particular, we are interested in dissecting endothelial cell signalling induced by the extracellular environment. For this we apply state-of-the-art mass spectrometry technology combined with SILAC-based quantitative approach.

You must hold an MD or a PhD in biology or a related field, be highly motivated, a team worker preferably with experience in the field of angiogenesis. Previous experience with mass spectrometry is not essential, but candidates should be willing to learn mass spectrometry based approaches and apply systems biology tools based on mass spectrometry to answer biological questions.

Applications with CV and names of three referees should be sent to Dr Sara Zanivan, by email to s.zanivan@beatson.gla.ac.uk.

\section{Ubiquitin Signalling - Dr. Danny Huang}

Our group is interested in understanding the molecular mechanism of histone ubiquitination and the functional roles of ubiquitin in transcription regulation. You will study the structure and function of ubiquitin machineries involved in histone modification. The laboratory is equipped with stateof-the-art equipment for protein expression, purification, crystallization, and X-ray diffraction.

You must have a relevant $\mathrm{PhD}$ and experience in protein purification, biochemical studies and protein structure determination by $\mathrm{X}$-ray crystallography.

Applications with CV and names of three referees should be sent to Dr Danny Huang, by email to d.huang@beatson.gla.ac.uk

\section{Checkpoint Signalling in Cancer Formation and Therapy - Prof David Gillespie}

The ATR-ChkI pathway is a key regulator of DNA damage responses and genome stability in vertebrate cells. You will join a team carrying out research to understand the role of the ATR-Chk I pathway in cancer formation, therapy, and tissue homeostasis using a combination of conventional and chemical genetic approaches. The project will involve the application of a wide range of cell, molecular, and biochemical techniques, including molecular cloning, gene targeting, flow cytometry, western blotting and protein kinase assays.

You must hold a relevant PhD and be highly motivated and proficient in standard molecular and cell biology techniques. Experience using genetically modified murine models would be an advantage.

Applications with CV and names of three referees should be sent to Prof. David Gillespie, by email to d.gillespie@beatson.gla.ac.uk

\section{Urology Research - Prof. Hing Leung}

The Urology Group focuses on how abnormal signalling drives human prostate cancer. Prostate cancer represents a major global health problem. Our programme of research applies cutting edge in vitro and in vivo methodology to understand cancer biology with an aim to develop novel targets for therapy in the context of personalised medicine.

You will join our vibrant group of researchers with basic and clinical scientists to investigate the key aberrant signalling in human prostate cancer focussing on receptor tyrosine kinases along with their regulatory factors and downstream signalling cascades. An example of possible network for investigation may involve the EGFR system and the Sprouty2 regulatory molecule and the downstream MAPK ERK5.

A relevant PhD is essential and you should be highly motivated and proficient in standard molecular and cell biology techniques. Experience in the in vitro and in vivo models, including transgenic models and proteomic methodologies would be an advantage.

Applications with CV and names of three referees should be sent to Prof. Hing Leung by email to h.leung@beatson.gla.ac.uk

If preferred applications may be sent to the relevant group leader by post to The Beatson Institute for Cancer Research, Garscube Estate, Switchback Road, Bearsden, Glasgow G6I IBD, UK.

Closing Date: | $8 / 1$ |/ |

\section{leuka}

The charity Leuka invites applications from the UK for funding research projects or PhD studentships related to leukaemia.

Potential applicants should in the first instance write briefly to Prof John Goldman, Chair of Leuka, Department of Haematology, Imperial College London, Du Cane Road, London W12 ONN.

UNIVERSITYOF BIRMINGHAM

Clinical Oncology MSc/PGDip/CPD We are training the elite oncologists of the future One year full-time, two years part-time, or week long CPD Further training is crucial for
keeping up to date with the lates issues in a rapidly changing world. Drawing on expertise of staff within the Cancer Research at the University of Birmingham as well as clinical staff from
Birmingham hospitals you wil study modules in:

- cancer biology

athology

- cancer therapy and

management

- clinical trials

- epidemiology and statistics
Optional modules include:

- haemato-oncology

- paediatric oncology

- palliative care

- caring for cancer patients

- radiation oncology

- radiation biology

a radiation physics

- translational research

- oncology surgery

The course is open to healthcare professionals and veterinary
specialists with at least six months experience in an
Full time students will benefit 6 month clinical observership as part of their programme. Learn more For further information including access to our on-line application form, please visit our webs www.mds.bham.ac.uk/ clinicaloncology or contact
Louise Batchelor on 0121414 7672 or email clinicaloncology @contacts.bham.ac.uk

www.mds.bham.ac.uk 


\section{Laboratoires Pierre Fabre}

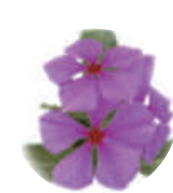

Santé familiale

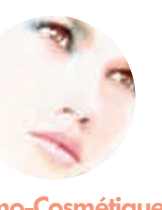

Dermo-Cosmétique

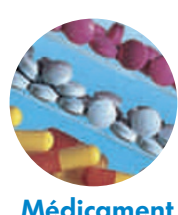

Médicament

\section{From Health to Beauty}

Research and innovation, ethical and pharmaceutical rigour, commitment to public health and first-class scientific

cosmetics... Based on these strong values, nurtured over more than forty years, Pierre Fabre Laboratories have developed, a global influence. Our principal strength: our 9,900 staff who share the same passion, rigour and imagination, comprising a company with sustainable growth and current sales of 1.86 billion euros in 130 countries.

An institute of excellence for biotechnology, the Center of Immunology Pierre Fabre (CIPF) is developing research programs in the field of cancer immunotherapy, in particular the identification of new targets and humanized therapeutic monoclonal antibodies. Inaugurated in 1990, the CIPF acquired its reputation through groundbreaking developments in the production of recombinant proteins and bacterial biomasses.

Position based in St-Julien-en-Genevois (France - Haute-Savoie)

Please apply on our website with a resume, cover letter and expected salary : www.pierre-fabre.com (in the section "Ressources Humaines - Offres en France Recherche et Développement" where you will find this vacancy) or send your application, with the reference I I539/MPU/NATURE to : Laboratoires Pierre Fabre, Direction du Développement des Compétences,

15 rue Théron Périé - 81106 Castres Cedex - France.

\section{Director of Antibody Engineering Research Department (M/F)}

Reporting directly to the Managing Director of the Center of Immunology Pierre Fabre, you will propose and implement research projects for the identification of new therapeutic antibodies. More specifically you will be in charge of:

- implementing experimental approaches adapted to projects in the field of antibody engineering and production,

ensuring scientific and competitive monitoring in the field of antibody engineering and more broadly in biotechnology,

contributing to the CIPF's visibility in the field of therapeutic antibodies by filling new patents,

publications and oral presentations,

- managing a research team of fifteen people.

$\mathrm{PhD}$. in Molecular Biology, you have a solid professional experience (minimum of 10 years) in Antibody Engineering within the pharmaceutical industry. You have gained international experience, proved by quality of publications, particularly in the field of antibodies. You master various experimental methods and you have prior experience in leading a research team. Good knowledge in drug discovery processes and operational methods of a pharmaceutical laboratory is required. Your english is fluent.

Your managerial and interpersonal skills allow you to interact easily with other research departments and you possess the results-driven mindset required for the position.

\section{Pierre Fabre \\ www.pierre-fabre.com}

\section{Four Year Postdoctoral Fellowships at}

\section{The London Research Institute Clare Hall \& Lincoln's Inn Fields Laboratories}

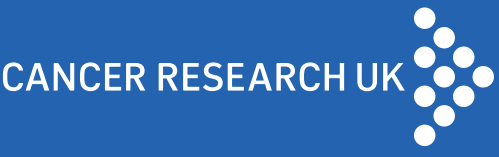

The London Research Institute (LRI) has an international reputation for innovative research into the basic biology of cancer and provides well-resourced research laboratories, core technology facilities and a four year Postdoctoral Programme. Our Research Group Leaders welcome enquiries from motivated and outstanding prospective Postdoctoral Fellows committed to a scientific career.

LRI Postdoctoral salaries start from $£ 30,970$ - $£ 36,739$ inclusive, plus pension benefits.

To find out more about our research, and postdoctoral opportunities at the LRI visit: http://www.london-research-institute.org.uk/jobs-education/postdoctoral-fellowships

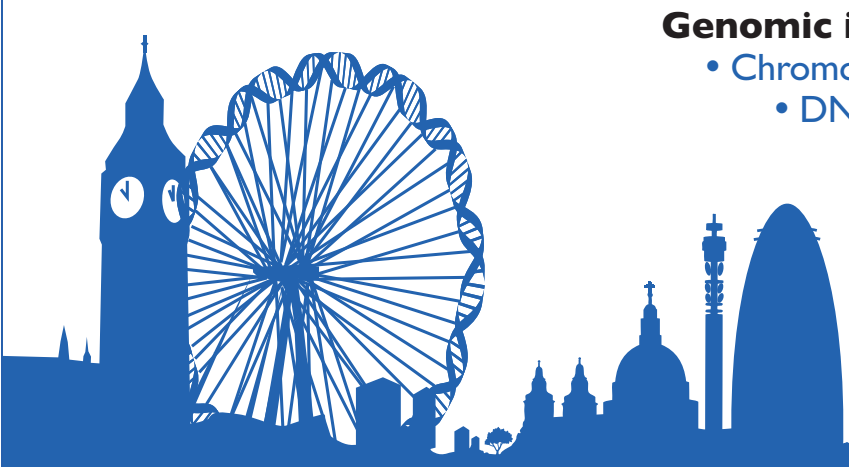

Genomic integrity and cell cycle

Chromosome biology • Checkpoint controls • DNA replication • - DNA damage and repair •

\section{Cellular regulatory mechanisms}

- Oncogenes and transformation • Signalling networks •

- Control of gene expression - Molecular cell biology

- Computational and structural biology •

\section{Biology of tissues and tumours}

- Tumour biology and cancer models • Vascular biology • -Immunology $\bullet$ Differentiation and growth control $\bullet$ - Metastasis • 


\section{Tenure/Tenure track faculty positions in Breast Cancer Indiana University School of Medicine, Indianapolis}

Indiana University School of Medicine is seeking applications for tenure/tenure track positions to establish a rigorous research program in breast cancer genomics and/or metastasis. The position includes an appointment as full-time faculty at a rank commensurate with experience in a basic science/or clinical department and is supported by an outstanding infrastructure, including a one teraFLOP/s supercomputer that has time dedicated to bioinformatics computing, a Biobank, $>2$ billion medical records, active genomics projects and a robust graduate training program. Applicants with active extramural funding and nationally/internationally recognized research program will be considered for an Endowed Chair. IU Simon Cancer Center is an $\mathrm{NCl}$ designated cancer center with breast cancer as a major program. The breast cancer program has 32 research and clinical faculty with research area ranging from understanding the basic biology of the normal breast to first-in-human clinical trials. The cancer center is also the home of Susan G. Komen normal breast tissue bank, which additionally stores serum, plasma, and DNA from healthy and breast cancer patients. The successful candidates are expected to establish independent research programs, collaborate with other investigators, and participate in the activities of the cancer center and the department.

Minimal qualifications for this position include an MD and/or PhD, post-doctoral experience for junior investigators, and a distinguished research/funding record for senior level position. The salary and recruitment package will be commensurate with the outstanding opportunity this represents.

Interested candidates should send: 1) a curriculum vitae; 2) contact information for five references; and 3) a 1-2 page statement outlining the applicant's research goals. Send all three as one PDF file by e-mail to Chasity Spears @ chasmill@iupui.edu with "Breast Cancer Position” as the subject of the message. The search and screen committee will begin reviewing applications as they are received and applications will be considered until an appointment is made.

Indiana University is an equal opportunity employer committed to building a culturally diverse intellectual community and strongly encourages applications from women and minorities.

\section{Mount Sinai}

Cancer Biology Positions at The Tisch Cancer Institute

The newly established Tisch Cancer Institute of Mount Sinai School of Medicine invites applications from outstanding scientists for faculty positions at the Assistant, Associate or Full Professor level. Our areas of interest include: Cancer model systems, Cancer epigenetics, Cancer stem cells, Cancer systems biology, Cancer therapeutics and Cancer immunology. Disease areas that are presently the focus of the Tisch Cancer Institute are: Hepatocellular carcinoma, Hematological malignancies, Head and neck cancer, Prostate, Breast, and Lung cancer.

Applicants should have an M.D., and/or Ph.D. degree with an outstanding record of publications. The successful candidate will receive generous startup resources with state-of-the-art laboratory space and institutional shared resources to support their research activities.

The Tisch Cancer Institute members are working together to integrate Mount Sinai's expanding research capacity. Over the next several years, the Cancer Institute will grow by 150,000 sq. ft. of research and clinical space in the new Center for Science and Medicine building. The Center for Science and Medicine is under construction with an expected occupancy by mid-2012.

Mount Sinai School of Medicine and Mount Sinai Hospital are among the world's leading biomedical institutions. The Medical Center is in the midst of a $\$ 1$ billion capital campaign in support of our $\$ 2.25$ billion strategic plan, which has a primary focus on the delivery of outstanding clinical care and translational research leading to therapeutic discoveries.

Candidates should send a CV, three letters of reference, and a summary of their research to: Steven Burakoff, M.D., Chair of the Search Committee, c/o Chan-Bene Lin (tci.recruitment@ mssm.edu) by October 31, 2011.

Mount Sinai Medical Center is an Equal Opportunity/Affirmative Action Employer. We recognize the power and importance of a diverse employee population and strongly encourage applicants with various experiences and backgrounds.

NW216219R

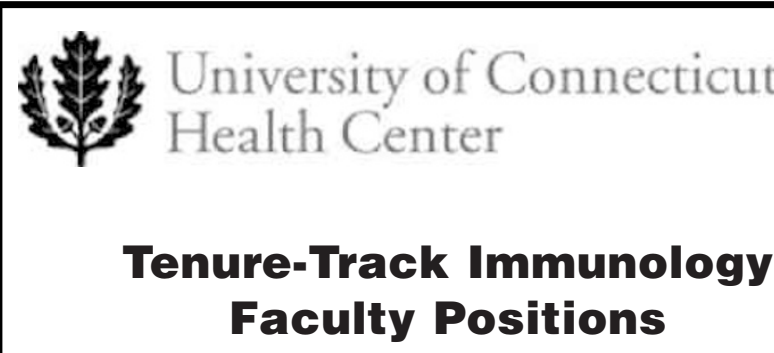

The Department of Immunology at the University of Connecticut Health Center seeks outstanding investigators for two tenuretrack positions at the Assistant/Associate/Full Professor level. Although all areas of immunology will be considered, we are particularly interested in individuals using molecular, cellular and translational approaches to study immune system function in vivo. Areas of priority include but are not limited to mucosal immunity, innate immunity, signal transduction and transcriptional control, and dendritic cell biology. The new hires will participate in a vibrant Ph.D. training program and have access to a growing translational research community. Salary and start-up funds are highly competitive and outstanding core facilities are available. Applicants must have a Ph.D. and/or M.D. with a history of sustained extramural funding and a high impact publication record. In addition to the beauty of the picturesque New England countryside, the Hartford area offers a vibrant arts and cultural scene and an exceptional outdoor sports environment. Applicants should apply at https://jobs.uchc.edu search number 2012-067 and submit curriculum vitae, a two-page summary of research interests and the names of three references. Information may also be submitted to Dr. Leo Lefrançois, Ph.D., Chairman, Department of Immunology, UConn Health Center, Farmington, CT. Email: immunology@uchc.edu. For further information on UCHC immunology, please visit http://immune.uchc.edu. 
The University of Utah Division of Hematology and Hematologic Malignancies in the Department of Medicine and Huntsman Cancer Institute (HCl) invite applications from physician-scientists at assistant or associate professor levels with a focus on hematologic malignancies. An emphasis on lymphoid malignancies or leukemia stem cell biology is desirable. The successful candidate will lead a translational program integrating laboratory-based research and clinical trials.

The Division of Hematology and Hematologic Malignancies currently has 24 faculty, including several internationally recognized leaders in the field. Well-developed research programs include myeloproliferative neoplasms and myeloma. The Division's hematopoietic stem cell transplant program performed more than 200 transplants in 2010. Our intention is to expand our clinical and research portfolio with an emphasis on translational malignant hematology. Significant resources will be made available to support recruitments. Division annual research funding is more than $\$ 7,000,000$.

The University of Utah is the primary research institution in the Intermountain West and hosts its only medical school. The University offers a breadth of research in an extremely collaborative environment. Unique resources include the Utah Population Database and ARUP laboratories, a nationally recognized diagnostic service within the framework of the University. The Department of Medicine is the largest department on the University of Utah campus, and has $\$ 65,000,000$ in annual NIH funding, exceeding by far most institutions of similar size.

$\mathrm{HCl}$ received renewal of its $\mathrm{NCl}$-Designated Cancer Center status in 2010; it is also a member of the National Comprehensive Cancer Network. $\mathrm{HCl}$ has a long-standing international reputation in cancer genetics. Several recent initiatives have placed top emphasis on translational and clinical research. These include the establishment of the Center for Investigational Therapeutics (a drug discovery and Phase I program), directed pilot funding, and new space and expansion of Huntsman Cancer Hospital. $\mathrm{HCl}$ is equipped with state-of-the-art laboratories and shared resources, including core facilities for imaging, genomics, drug screening, and population studies. We offer strong institutional support, a collegial and interactive research environment that fosters the development of junior faculty, and robust graduate programs for training PhD and MD/PhD students. Visit www.utahinternalmedicine.org and www.huntsmancancer.org for more information.

Submit a curriculum vitae and a description of research interests and accomplishments with three letters of recommendation online at http://utah.peopleadmin.com/postings/9617. Applications accepted until positions filled.

The University of Utah values candidates who have experience working in settings with students from diverse backgrounds and possess a strong commitment to improving access to higher education for historically underrepresented students.

The University of Utah is an Equal Opportunity/Affirmative Action employer and educator. Minorities, women, and persons with disabilities are strongly encouraged to apply. Veterans' preference. Reasonable accommodations provided. For additional information: http://www.regulations.utah.edu/humanResources/5-106.html.

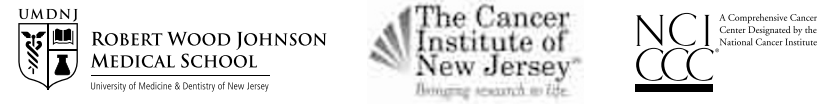

\section{FACULTY POSITIONS AVAILABLE Cancer Research}

The Cancer Institute of New Jersey (CINJ) invites applications for tenuretrack faculty positions specializing in cancer progression, genetic instability, metastasis, genetics, metabolism, signaling or microenvironment. Exceptional senior investigators will also be considered. $\mathrm{CINJ}$ is an $\mathrm{NCl}$-designated, comprehensive and consortium cancer center that includes faculty members from UMDNJ-Robert Wood Medical School, Rutgers and Princeton Universities.

CINJ website http://www.cinj.org/

New faculty are expected to establish an innovative, independent and collaborative research program addressing important, fundamental questions in the area of cancer biology. Interdisciplinary approaches are encouraged and the combined $\mathrm{CINJ}$ consortium faculty has complementary expertise and strength in cancer biology, signal transduction, systems biology, metabolism, and genomic instability. Qualified candidates must have a Ph.D. and/or M.D., or equivalent graduate degree, and outstanding academic credentials. We seek candidates who are independent investigators interested in collaboration with basic scientists and with clinical faculty for translational research. Rank will be commensurate with experience.

Interested applicants should send their CV, a brief description of their research interests and plans, and the names of three references via email to cinjrecruitment@gmail.com addressed to: Search Committee, c/o Larissa Varela, Faculty Recruitment Office, Cancer Institute of New Jersey, 120 Albany St, Tower 2, 5th Floor, New Brunswick, NJ, 08901. Email: cinjrecruitment@gmail.com. UMDNJ is an EEO/AA employer, $\mathrm{m} / \mathrm{f} / \mathrm{h} / \mathrm{v}$, and a member of the University Health System of New Jersey

\section{Yale cancer \\ OIV CENTER A Comprestiensine Cancor Centar Designated} by the National Cancer Institute

YALE-NEW HAVEN HOSPITAL

\section{Yale Cancer Center Cancer Biology Institute}

The Yale Comprehensive Cancer Center, its brand new Cancer Biology Institute, and the Yale School of Medicine invite applications from basic science investigators for junior or senior appointments with interests in cancer biology, in areas including cancer genetics and genomics, signal transduction, structural biology, proteomics, mass spectrometry and drug discovery. The Cancer Biology Institute is one of 5 newly formed multidisciplinary research institutes at Yale's West Campus, a 136 acre parcel located 7 miles from Yale's New Haven campus that includes 20 buildings and 1.6 million square feet of research, office, and warehousing space. Additional West Campus assets include the Institute of Chemical Biology, Institute of Microbial Diversity, Institute of Systems Biology, Institute of Biodesign, as well as core facilities in high throughput cell biology, small molecule discovery, and genomic analysis. The position will have a role in defining and implementing the vision for cancer biology research at Yale going forward. This includes developing translational research opportunities with the recently opened Smilow Cancer Hospital at YaleNew Haven. Appointments at Yale Medical School and at Yale faculty of Arts and Sciences are available in a number of departments and will be commensurate with a demonstrated record of scholarly achievement. Of particular importance is experience in one or more of the following; a record of original research in cancer biology, independent extramurally funded laboratory investigation for senior applicants, engagement in translational research activities, and/or the development of strong cooperative teams across disciplines. Women and minority candidates are urged to apply.

Please submit a letter describing qualifications, with a CV and three letters of reference by [30 days from publication date] to: Dr. Joseph Schlessinger, Director, Cancer Biology Institute, Yale Cancer Center, c/o Vickie Johnson, 333 Cedar St., PO Box 208028, New Haven, CT 065208028. Yale University is an Equal Opportunity/Affirmative Action Employer. 


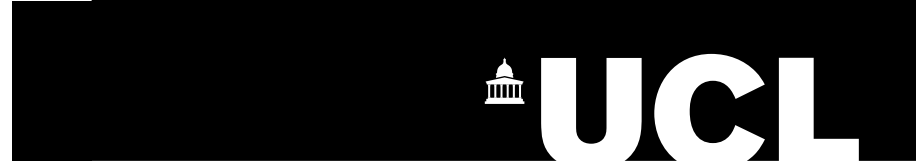

UCL Institute of Child Health

Molecular Haematology and Cancer Biology

\section{Lecturer / Senior Lecturer}

Applications are invited for a new full time post of Lecturer / Senior Lecturer in Epigenetics and Cancer Biology in the Molecular Haematology and Cancer Biology Unit. This is a key strategic appointment to establish and develop a research programme, provide leadership for and build capacity in epigenetics research.

If the appointment is made at Lecturer level the salary will be Grade 8 - £39,668 - £46,822 per annum. If the appointment is made at Senior Lecturer level the salary will be Grade 9 - £50,902 - £55,362 per annum depending on skills and experience.

The salaries include London Allowance.

A central theme in the MHCB Unit is the investigation of the basic mechanisms of gene regulation in the context of normal tissue development and how alterations in the pathways that control cell proliferation, growth, differentiation, cell survival or the immune response contribute to the development and maintenance of childhood cancers. We would like to complement this research by recruiting a new group leader to work on chromatin or genome regulation.

The Lecturer or Senior Lecturer will be expected to establish an innovative research programme that contributes epigenetics expertise and methodological strengths to existing and emerging programmes of research within the Cancer Theme.

An interest in studying epigenetic regulation of the genome during normal development of the haematopoietic system or the peripheral and central nervous systems, and in the major childhood tumours of these tissues - leukaemias, neuroblastoma, brain tumours - would be particularly welcome. Requirements for appointment at Senior Lecturer are identified within the person specification.

For further details about the vacancy and how to apply online please go to http://www.ucl.ac.uk/hr/jobs/ and search on Reference Number 1204133.

Informal enquiries about this position may be made to Dr. Jonathan Ham, email: j.ham@ich.ucl.ac.uk

If you have any queries regarding the vacancy or the application process, please contact Daniel Henniker on email:

d.henniker@ich.ucl.ac.uk, tel: 02079052742 quoting job reference 1204133

We particularly welcome female applicants and those from an ethnic minority, as they are under-represented within UCL at this level.

UCL Taking Action for Equality

\section{Closing date for applications: 17 October 2011}

UCL Institute of Child Health in partnership with Great Ormond Street Hospital for Children NHS Trust

\section{naturejobs}

Read our Postdoc articles and news in the

News and Comment section of naturejobs.com

- Helpful advice

- Real life experiences

- Follow the highs and lows of postdoc life

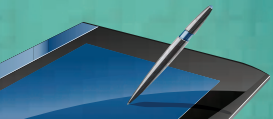

Job Title: BONE MARROW TRANSPLANTATION POST-DOCTORAL SCIENTIST

Job Description:

A world-renowned laboratory studying the biology of graft versus host disease and graft versus leukemia in the Blood and Marrow Transplant Program at the University of Michigan is recruiting a highly motivated, experienced postdoctoral fellow to join a closely integrated team that is studying proteomic and cellular biomarkers for complications after hematopoietic stem cell transplantation.

\section{Requirements:}

- $\mathrm{PhD}$ and/or $\mathrm{MD}$ with substantial research experience in immunology and cell biology

- Demonstrated technical independence with flow cytometry (at least 6 colors with Flowjo software knowledge), $T$ cells and dendritic cells culture and functional assays, and immunoassays (ELISPOT/ELISA), and/or confocal microscopy

- Meticulous at planning, executing, and organizing the results for large scale assays.

- S/he must also be capable of taking initiative, work independently and in a collaborative setting

- A good publication record in recognized peer-reviewed journals

- Excellent written and verbal (English) communication skills are required, as are excellent organizational skills and knowledge of proper scientific record keeping

Compensation: Competitive salary + excellent benefits

Applicants should send an updated CV including publication list and two academic reference letters to:

Sophie Paczesny, MD, PhD

Assistant Professor Blood and Marrow Transplant Program University of Michigan Comprehensive Cancer Center 6410 Cancer Center

1500 E. Medical Center Dr., SPC 5942

Ann Arbor, MI 48109-5942

Email: sophiep@umich.edu

NW216704R

\section{Stay in touch with the latest scientific news and research, anytime, anywhere}

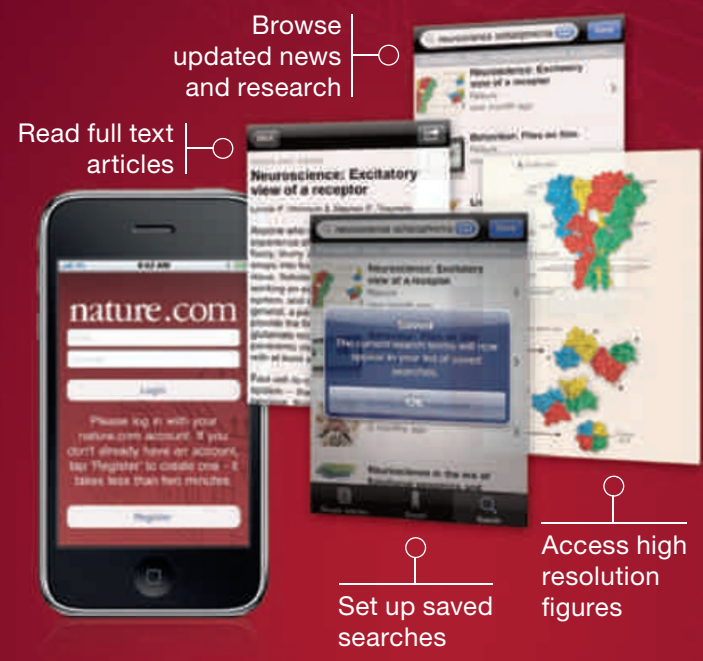

With the new nature.com mobile app you can read scholarly content quickly and comfortably wherever you are 


\section{Accelerating Science: Concept to Clinic}
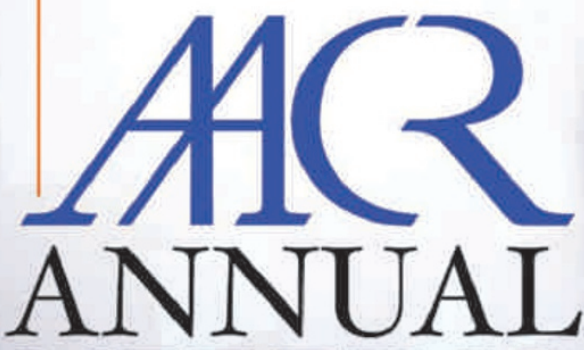

MEETING

2012

March 31- April 4

McCormick Place West

Chicago, IL

Don't miss the premier forum

for the latest breakthroughs in cancer research, bringing together over 17,000 attendees from over 60 countries. More than 6,000 proffered papers and hundreds of invited talks from leading experts will be presented, covering the full spectrum of cancer research, including basic, translational, clinical, and population research.

New in 2012:

- A revised format for the presentation of accepted phase II and III clinical trials

- New session series titled Concepts and Controversies in Diagnostics, Therapeutics, and Prevention

- First-ever poster session titled Clinical Trials in Progress

- A regulatory track with sessions on regulatory science and science policy

Chairperson: Benjamin G. Neel, Ontario Cancer Institute, Princess Margaret Hospital, University of Toronto, Toronto, ON, Canada

\section{AC2 American Association for Cancer Research www.aacr.org/annmeeting12}

Abstract Submission Deadline: Tuesday, November 15

Early Registration Deadline: Thursday, December 22

Late-breaking and placeholder abstract submission deadline: Wednesday, January 25 